\title{
Variabilidade espacial de MO, P, K e CTC do solo sob diferentes usos e manejos
}

\author{
Spatial variability of MO, P, K and CTC of soil under different use and management conditions
}

\author{
Eloiza Gomes Silva Cavalcante ${ }^{\mathrm{I}}$ Marlene Cristina Alves ${ }^{\mathrm{I}}$ Gener Tadeu Pereira $^{\mathrm{II}}$ \\ Zigomar Menezes de Souza ${ }^{\text {III }}$
}

\section{RESUMO}

O estudo da variabilidade espacial dos atributos químicos dos solos é particularmente importante em áreas onde o solo está submetido a diferentes manejos. O trabalho foi desenvolvido em Selvíria (MS), com o objetivo de avaliar a variabilidade espacial da matéria orgânica (MO), o fósforo (P), do potássio (K) e a capacidade de troca catiônica (CTC) em um Latossolo Vermelho distrófico sob diferentes usos e manejos. Os solos foram amostrados em uma malha, com intervalos regulares de $2 \mathrm{~m}$, perfazendo um total de 64 pontos, nas profundidades de 0,0-0,1m e 0,1-0,2m, nas seguintes áreas: vegetação natural (cerrado), plantio direto, plantio convencional e pastagem. O sistema de plantio direto apresentou acúmulo significativo de matéria orgânica, fósforo, potássio e elevação da CTC em relação aos demais sistemas estudados, além da melhoria nas condições químicas do solo. A matéria orgânica foi maior em relação ao sistema natural. As maiores variabilidades medidas por meio do coeficiente de variação foram observadas para o fósforo e o potássio, sendo que a matéria orgânica e a CTC apresentaram coeficiente de variação médio nos diferentes usos e manejos do solo.

Palavras-chave: atributos químicos do solo, preparo do solo, geoestatística, latossolo.

\section{ABSTRACT}

The study of the spatial variability of the chemical attributes of the soils is particularly important in areas where the soil is submitted to different management. This work was developed in Selvíria (MS) with the objective of evaluating the spatial variability of the organic matter (MO), phosphorus $(P)$, potassium (K) and cation exchange capacity (CEC) in a oxisol under different use and management conditions. The soils were collected in a grid, with regular intervals of $2 \mathrm{~m}$, total of 64 points, in the depths of 0,0-0,1m and 0,1-0,2m, in the use and management were: native vegetation (savannah), no-tillage, conventional system and pasture. The system of no-tillage presented significant accumulation of organic matter, phosphorus, potassium and elevation of CEC in relation to the other studied systems, besides the improvement in the chemical conditions of the soil, the organic matter was larger in relation to the natural system. The largest variabilities measured through the variation coefficient, they were observed for the phosphorus and potassium, and the organic matter and CEC presented medium variation coefficient in the different uses and management of the soil.

Key words: chemical attributes of soil, soil tillage, geostatistics, oxisol.

\section{INTRODUÇÃO}

Pela própria natureza dos fatores responsáveis pela sua formação, o solo apresenta heterogeneidade, tanto vertical como horizontalmente. Este fato ocorre porque o próprio material de origem não é uniforme em toda a sua extensão, ou seja, o processo de intemperização não ocorre de forma homogênea e contínua. Segundo os princípios básicos da experimentação, a variabilidade do solo ocorre de forma aleatória. No entanto, várias pesquisas mostram que a variabilidade dos atributos químicos do solo

IDepartamento de Fitossanidade, Engenharia Rural e Solos, Faculdade de Engenharia (FE), Campus de Ilha Solteira, Universidade Estadual Paulista (UNESP), CP 31, 15385-000, Ilha Solteira, SP, Brasil. E-mail:cavalcante@agr.feis.unesp.br. *Autor para correspondência.

IIDepartamento de Ciências Exatas, Faculdade de Ciências Agrárias e Veterinárias (FCAV), UNESP, Jaboticabal, SP, Brasil.

IIIFaculdade de Engenharia Agrícola (FEAGRI), Universidade de Campinas (UNICAMP), Campinas, SP, Brasil. 
apresenta correlação ou dependência espacial (TRANGMAR et al., 1985; SOUZA et al., 1997; OLIVEIRA et al., 1999; CARVALHO et al., 2002).

Na atualidade a fertilização é efetuada em grandes áreas consideradas homogêneas, considerando a necessidade média para a aplicação de insumos (fertilizantes, defensivos, água, etc.), fazendo com que a mesma formulação e/ou quantidade dos fertilizantes seja utilizada para toda a área, atendendo apenas as necessidades médias e não considerando, dessa forma, as necessidades específicas de cada parte do campo. O mesmo acontece para os demais insumos, o que resulta em uma lavoura com produtividade nãouniforme (TSCHIEDEL \& FERREIRA, 2002). Esta mudança é necessária para que se entenda a propriedade não homogênea e que se trate cada parte conforme sua necessidade, fazendo com que o produtor tenha o conhecimento detalhado de sua propriedade.

O uso de insumos químicos agrícolas tem sido identificado como o principal fator de contaminação da água e do solo (BHATTI et al., 1991). As aplicações convencionais de fertilizantes e defensivos significam aplicações excessivas em algumas áreas de campo e insuficiente em outras, pois uma área nunca é totalmente uniforme, por menor que seja. O uso da geoestatística, para modelar a variabilidade espacial do solo, permite a aplicação de insumos no local correto e na quantidade exata, promovendo o aumento da produtividade das culturas e diminuindo grandemente o risco de contaminações ambientais, para áreas cada vez menores e mais homogêneas, tanto quanto a tecnologia e os cultos envolvidos o permitam.

O estudo da variabilidade espacial dos atributos químicos dos solos é particularmente importante em áreas onde o solo está submetido a diferentes manejos (CARVALHO et al., 1998; CARVALHO et al., 2002), pois a análise geoestatística pode indicar alternativas de manejo não só para reduzir os efeitos da variabilidade na produção das culturas (TRANGMAR et al., 1985; BHATTI et al., 1991), mas também para aumentar a possibilidade de se estimarem respostas de atributos químicos do solo em função de determinadas práticas de manejo (OVALLES \& REY, 1994; SILVEIRA et al., 2000). Além disso, a estratégia de amostragem do solo pode ser melhorada com a incorporação de um modelo de variabilidade espacial (WILDING \& DREES, 1983; CARVALHO et al., 1992).

Nesse sentido, desenvolveu-se este trabalho com o objetivo de estudar a variabilidade espacial de alguns atributos químicos de um Latossolo Vermelho sob diferentes usos e manejos.

\section{MATERIAL E MÉTODOS}

O estudo foi realizado na Fazenda de Ensino e Pesquisa da Faculdade de Engenharia, Campus de Ilha Solteira, da Universidade Estadual Paulista "Júlio de Mesquita Filho", situada à margem direita do rio Paraná, no município de Selvíria (MS), localizado nas coordenadas geográficas $20^{\circ} 22^{\prime} \mathrm{S}$ e $51^{0} 22^{\prime} \mathrm{W}$, numa altitude média de $335 \mathrm{~m}$. O tipo climático, segundo Köppen, é Aw, caracterizado como tropical úmido com estação chuvosa no verão e seca no inverno, estando a umidade relativa dos meses mais chuvosos entre 60 e $80 \%$. O solo foi classificado como Latossolo Vermelho distrófico típico argiloso A moderado hipodistrófico, álico, caulinítico, férrico, compactado, muito profundo, moderadamente ácido ( $\mathrm{LVd})$.

A área com vegetação natural, onde predominam as formas arbustivas em solo profundo e pouco fértil, conhecida como cerrado, foi desmatada em 1978, sendo, em 1990, instalado o sistema de plantio direto, em 1980, o preparo convencional e a pastagem em 1978. O plantio direto apresenta uma seqüência de culturas com leguminosas e gramíneas, tais como feijão (Phaseolus vulgaris), milho (Zea mays L.), soja (Glycine Max (L) Merril) e aveia preta (Avena strigosa Schieb), enquanto o manejo de plantas invasoras tem sido efetuado com herbicidas, sendo que a área de plantio direto vem sendo cultivada com este sistema há oito anos. O preparo convencional consta de gradagem pesada (aradora) e gradagem leve (niveladora), com cultivo de culturas anuais. O cultivo desta área encontrava-se com 18 anos. Efetuou-se a calagem antes da instalação dos sistemas de plantio direto e preparo convencional, com o objetivo de elevar a saturação por bases a $60 \%$. As culturas de feijão, milho e soja receberam uma adubação anual de $200 \mathrm{~kg}$ $\mathrm{ha}^{-1}$ da formula 4-30-10, enquanto a área de pastagem (Brachiaria decumbens) só recebeu calagem e adubação na implantação e nas reformas esta área possui 20 anos de cultivo.

A amostragem dos atributos químicos do solo estudado foi feita nos pontos de cruzamento de uma malha regular, com intervalos regulares de $2 \mathrm{~m}$, perfazendo um total de 64 pontos (14 x 14), sendo coletada uma amostra por ponto (Figura 1), nas profundidades de $0,0-0,1 \mathrm{~m}$ e $0,1-0,2 \mathrm{~m}$. Os valores das variáveis fósforo e potássio disponíveis foram extraídos utilizando o método da resina trocadora de íons proposta por RAIJ et al. (2001). Com base nos resultados das análises químicas, foram calculados os valores da variável capacidade de troca catiônica (CTC). A determinação dos teores de matéria orgânica foi realizada segundo metodologia proposta por RAIJ et al. (2001).

Ciência Rural, v.37, n.2, mar-abr, 2007. 


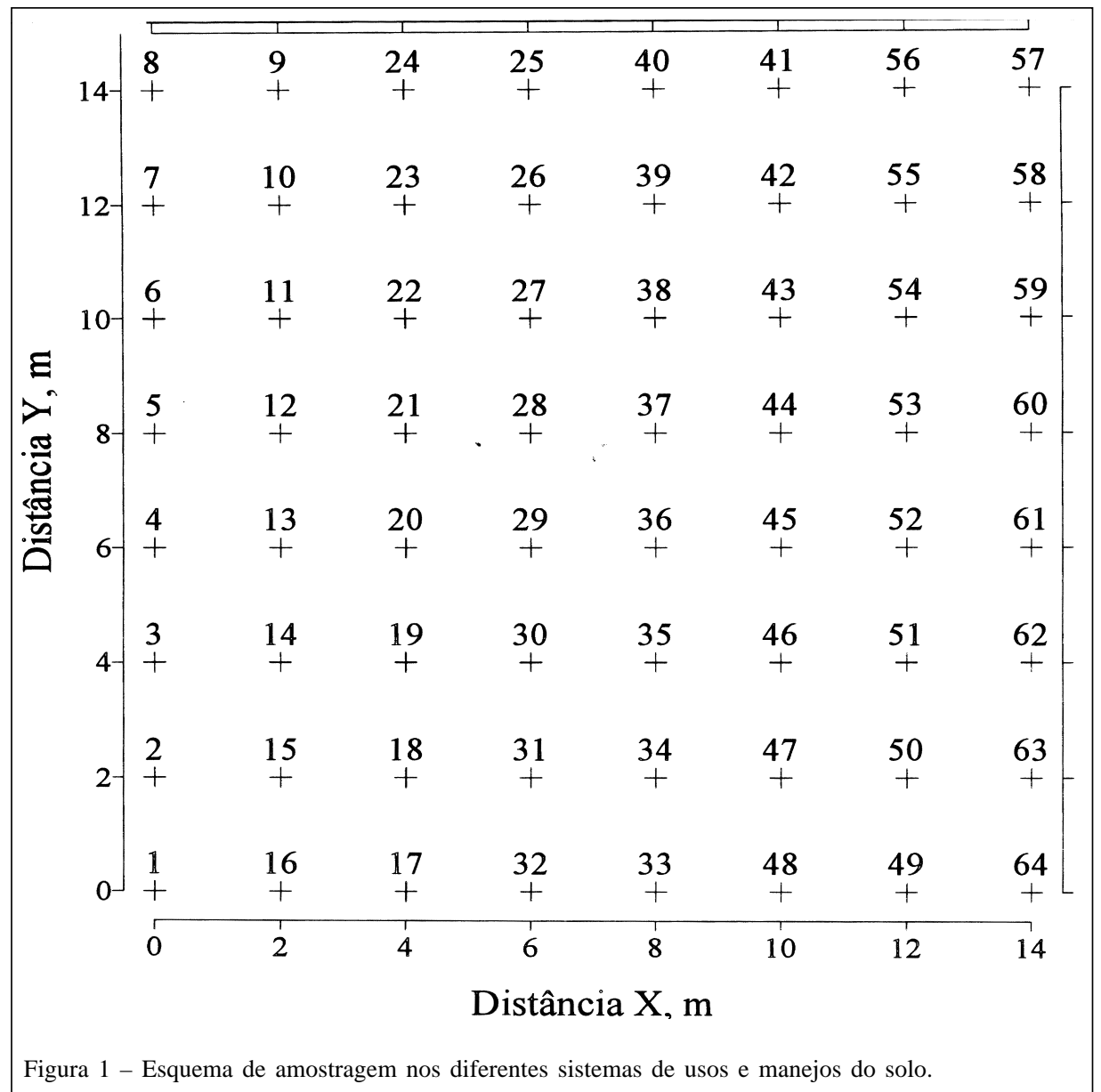

Os atributos químicos do solo foram analisados por meio da análise estatística descritiva e de técnicas geoestatísticas. A hipótese de normalidade dos dados foi testada pelo teste de KolmogorovSmirnov, SAS (SCHLOTZHAVER \& LITTELL, 1997). A dependência espacial foi analisada por meio de ajustes de semivariogramas (VIEIRA et al., 1983; ROBERTSON, 1998), com base na pressuposição de estacionariedade da hipótese intrínseca, a qual é estimada por:

$$
\hat{\gamma}(h)=\frac{1}{2 N(h)} \sum_{i=1}^{N(h)}\left[Z\left(x_{i}\right)-Z\left(x_{i}+h\right)\right]^{2}
$$

em que $\mathrm{N}$ (h) é o número de pares experimentais de observações $\mathrm{Z}\left(\mathrm{x}_{\mathrm{i}}\right)$ e $\mathrm{Z}\left(\mathrm{x}_{\mathrm{i}}+\mathrm{h}\right)$ separados por uma distância h. O semivariograma é representado pelo gráfico $\hat{\gamma}(h)$ versus h. Do ajuste de um modelo matemático aos valores calculados de , são estimados os coeficientes do modelo teórico para o semivariograma (o efeito pepita, $\mathrm{C}_{0}$; patamar, $\mathrm{C}_{0}+\mathrm{C}_{1}$; e o alcance, a). Os modelos de semivariogramas considerados foram o esférico, o exponencial, o linear e o gaussiano (McBRATNEY \& WEBSTER, 1986). Para analisar o grau da dependência espacial dos atributos químicos em estudo, utilizou-se a classificação de CAMBARDELLA et al. (1994), em que são considerados de dependência espacial forte os semivariogramas que têm um efeito pepita $=25 \%$ do patamar, moderada quando está entre 25 e $75 \%$, e fraca $>75 \%$.

Estes modelos foram ajustados através do programa GS+ (ROBERTSON, 1998). Em caso de dúvida entre mais de um modelo para o mesmo semivariograma, considerou-se o maior valor do coeficiente de correlação obtido pelo método de validação cruzada e menor SQR (soma de quadrados do resíduo).

\section{RESULTADOS E DISCUSSÃO}

Os maiores valores de $\mathrm{P}$, $\mathrm{K}$ e CTC foram observados no sistema de plantio direto e a pastagem com os menores valores para estes atributos nas profundidades estudadas (Tabela 1). Resultados semelhantes foram verificados por SOUZA \& ALVES (2003), estudando os atributos químicos de um Latossolo Vermelho distrófico de cerrado sob diferentes 
Tabela 1 - Estatística descritiva para as variáveis matéria orgânica $\left(\mathrm{g} \mathrm{dm}^{-3}\right)$, fósforo $\left(\mathrm{mg} \mathrm{dm}^{-3}\right)$, potássio $\left(\mathrm{mmol}_{\mathrm{c}} \mathrm{dm}^{-3}\right)$ e capacidade de troca catiônica $\left(\mathrm{mmol}_{\mathrm{c}} \mathrm{dm}^{-3}\right)$ de amostras coletadas na malha nas profundidades de 0,0-0,1 m e 0,1-0,2 m.

\begin{tabular}{|c|c|c|c|c|c|c|c|c|}
\hline \multirow{2}{*}{ Estatística } & \multicolumn{4}{|c|}{ Profundidade de $0,0-0,1 \mathrm{~m}$} & \multicolumn{4}{|c|}{ Profundidade de $0,1-0,2 \mathrm{~m}$} \\
\hline & MO & $\mathrm{P}$ & $\mathrm{K}$ & CTC & MO & $\mathrm{P}$ & $\mathrm{K}$ & СТC \\
\hline \multicolumn{9}{|c|}{ Cerrado } \\
\hline Média & 30,6 & 6,9 & 0,9 & 57,0 & 19,6 & 4,3 & 0,3 & 45,1 \\
\hline Mediana & 31,0 & 7,0 & 1,0 & 57,0 & 19,0 & 4,0 & 0,2 & 42,0 \\
\hline Desvio padrão & 4,9 & 1,4 & 0,0 & 6,8 & 2,9 & 10,6 & 0,4 & 5,1 \\
\hline${ }^{1} \mathrm{CV}$ & 16,1 & 25,8 & 30,5 & 12,2 & 14,9 & 24,8 & 67,2 & 12,3 \\
\hline Assimetria & 0,2 & 0,6 & 0,7 & 0,6 & 2,6 & 0,6 & 6,3 & 1,2 \\
\hline Curtose & 2,9 & 3,5 & 2,6 & 4,0 & 8,1 & 3,9 & 16,1 & 4,8 \\
\hline${ }^{2} \mathrm{~d}$ & $0,09^{*}$ & 0,2 & 0,2 & $0,09^{*}$ & 0,2 & 0,2 & 0,4 & 0,2 \\
\hline \multicolumn{9}{|c|}{ Plantio direto } \\
\hline Média & 42,4 & 34,5 & 4,6 & 108,0 & 30,0 & 12,0 & 1,8 & 82,9 \\
\hline Mediana & 42,0 & 32,0 & 4,2 & 106,0 & 30,0 & 12,0 & 1,7 & 82,5 \\
\hline Desvio padrão & 0,4 & 10,0 & 0,2 & 1,3 & 3,1 & 3,5 & 0,8 & 7,4 \\
\hline $\mathrm{CV}$ & 13,6 & 29,2 & 45,1 & 12,5 & 12,4 & 28,9 & 46,9 & 12,7 \\
\hline Assimetria & 0,6 & 0,5 & 1,5 & 0,3 & 0,5 & $-0,2$ & 0,5 & 0,0 \\
\hline Curtose & 4,2 & 3,0 & 5,6 & 3,1 & 3,2 & 2,5 & 2,6 & 2,5 \\
\hline $\mathrm{d}$ & 0,1 & 0,1 & $0,07^{*}$ & $0,09^{*}$ & 0,2 & 0,1 & $0,08^{*}$ & $0,08^{*}$ \\
\hline \multicolumn{9}{|c|}{ Preparo convencional } \\
\hline Média & 23,6 & 17,0 & 1,5 & 62,6 & 22,6 & 11,0 & 0,4 & 55,8 \\
\hline Mediana & 24,0 & 16,0 & 1,4 & 62,0 & 22,0 & 9,0 & 0,3 & 53,0 \\
\hline Desvio padrão & 2,7 & 9,0 & 0,5 & 9,4 & 3,1 & 3,9 & 0,4 & 6,2 \\
\hline $\mathrm{CV}$ & 12,4 & 52,3 & 39,1 & 15,0 & 14,6 & 35,0 & 58,8 & 12,8 \\
\hline Assimetria & $-0,1$ & $-0,2$ & 1,1 & 1,4 & 0,0 & 0,6 & 1,7 & 0,5 \\
\hline Curtose & 1,8 & 12,8 & 4,1 & 5,6 & 3,1 & 2,6 & 5,5 & 2,8 \\
\hline d & 0,2 & 0,3 & 0,4 & 0,2 & 0,2 & 0,1 & 0,3 & $0,09^{*}$ \\
\hline \multicolumn{9}{|c|}{ Pastagem } \\
\hline Média & 23,0 & 10,0 & 0,6 & 46,5 & 17,5 & 4,6 & 0,1 & 39,8 \\
\hline Mediana & 23,0 & 9,0 & 0,5 & 47,0 & 18,0 & 4,0 & 0,1 & 40,0 \\
\hline Desvio padrão & 3,5 & 4,7 & 0,7 & 4,5 & 2,2 & 2,4 & 0,0 & 12,4 \\
\hline $\mathrm{CV}$ & 15,3 & 47,1 & 76,8 & 12,7 & 12,7 & 51,2 & 74,2 & 13,5 \\
\hline Assimetria & 0,6 & 1,3 & 2,5 & 0,0 & 0,6 & 1,7 & 2,5 & 0,3 \\
\hline Curtose & 3,0 & 4,9 & 12,8 & 2,6 & 4,6 & 6,1 & 8,4 & 3,1 \\
\hline d & 0,2 & 0,1 & 0,4 & 0,2 & 0,2 & 0,2 & 0,4 & $0,09^{*}$ \\
\hline
\end{tabular}

${ }^{1}$ - CV = coeficiente de variação; ${ }^{2}$ - d = estatística do teste de Kolmogorov-Smirnov, significativo a 5\% de probabilidade $\left({ }^{*}\right)$.

usos e manejos. Os sistemas de plantio direto e convencional apresentaram os maiores valores de $\mathrm{P}, \mathrm{K}$ e CTC quando comparados aos sistemas de cerrado e pastagem, uma vez que os dois primeiros sistemas envolviam o uso de culturas anuais, nas quais foram feitas adubações com fertilizantes minerais que continham esses nutrientes.

Observou-se acúmulo de P e K na superfície, bem como a diminuição dos teores em profundidade para todos os sistemas estudados, o que também foi verificado por vários pesquisadores (CENTURION et al., 1985; ELTZ et al., 1989; BAYER \& MIELNICZUK, 1997; SOUZA \& ALVES, 2003). Tal comportamento é explicado, segundo os autores, principalmente nos sistemas de plantio direto, pelo não-revolvimento, favorecendo o acúmulo de nutrientes na superfície e, no caso do fósforo, pela sua baixa mobilidade no solo (CENTURION et al., 1985; DE MARIA \& CASTRO, 1993; SILVEIRA \& STONE, 2001). Os sistemas de preparo convencional e pastagem apresentaram uma redução substancial nos teores de MO em relação aos demais, provavelmente pelo uso de manejo inadequado.

Comparando os diferentes sistemas, observa-se que os sistemas de cerrado e plantio direto apresentaram os maiores teores de MO em relação aos sistemas de preparo convencional e pastagem nas profundidades estudadas (Tabela 1). Constatou-se uma diminuição dos teores de MO em profundidade para 
todos os sistemas de usos e manejos estudados. Resultados semelhantes foram observados por CENTURION et al. (1985), DERPSCH et al. (1991) e SOUZA\& ALVES (2003). A diminuição do teor de MO nos cultivos contínuos pode ser atribuída à decomposição da MO humificada em virtude do baixo retorno dos resíduos vegetais ao solo.

Os resultados referentes à análise descritiva para os teores de MO, P, K e CTC são apresentados na tabela 1. Nota-se que os valores da média e mediana de todas as variáveis são próximos, caracterizando distribuição simétrica. Somente as variáveis MO no sistema de cerrado, K no sistema de plantio direto, CTC nos sistemas de cerrado, plantio direto na profundidade 0,0-0,1m, K no sistema de plantio direto, CTC nos sistemas de plantio direto, preparo convencional e pastagem na profundidade de $0,1-0,2 \mathrm{~m}$ apresentaram não-significância pelo teste Kolmogorov-Smirnov. A normalidade dos dados não é uma exigência da geoestatística, é conveniente apenas que a distribuição não apresente caudas muito alongadas, o que poderia comprometer as estimativas da krigagem, as quais são baseadas nos valores médios (ISAAKS \& SRIVASTAVA, 1989; WARRICK \& NIELSEN, 1980). Mais importante que a normalidade dos dados é a ocorrência do efeito proporcional em que a média e a variância dos dados não sejam constantes na área de estudo, fato este que não ocorreu no presente estudo, tendo em vista que os semivariogramas apresentaram patamares bem definidos.

De acordo com a classificação do coeficiente de variação (CV) proposta por WARRICK \& NIELSEN (1980), as variáveis MO e CTC nas profundidades de 0,0-0,1m e 0,1-0,2m apresentaram CV médio (12 a 24\%) em todos os sistemas de usos e manejos estudados (Tabela 1). Resultados semelhantes foram encontrados por SOUZA et al. (1997), ARAUJO (2002) e SOUZA et al. (2003) para a variável MO. As variáveis $\mathrm{P}$ e $\mathrm{K}$ nas profundidades estudadas apresentaram CV alto (> 24\%) em todos os sistemas de usos e manejos estudados, concordando com os resultados obtidos por SOUZA et al. (1997), SALVIANO et al. (1998), SILVEIRA et al. (2000) e CARVALHO et al. (2002).

Os resultados da análise geoestatística (Tabela 2) mostraram que todas as variáveis analisadas apresentaram dependência espacial nas duas profundidades estudadas, com exceção da variável $\mathrm{K}$ no sistema de pastagem na profundidade de $0,1-0,2 \mathrm{~m}$, a qual apresentou efeito pepita puro, indicando, segundo CAMBARDELLA et al. (1994), uma variabilidade não explicada, que pode ser devida a erros de medida e microvariações não detectadas, considerando a distância de amostragem utilizada. Todos os semivariogramas ajustaram-se aos modelos esférico e exponencial, exceção feita para a variável MO no sistema de preparo convencional em ambas as profundidades estudadas, $\mathrm{P}$ no sistema de pastagem na profundidade de $0,0-0,1 \mathrm{~m}$, K nos sistemas de cerrado e plantio direto nas profundidades de $0,0-0,1 \mathrm{~m}$ e 0,1 $0,2 \mathrm{~m}$, respectivamente, e CTC no sistema de plantio direto nas profundidades estudadas que se ajustaram ao modelo linear.

Na análise do grau de dependência espacial dos atributos químicos em estudo, utilizou-se a classificação de CAMBARDELLA et al. (1994). A relação $\mathrm{C}_{0} /\left(\mathrm{C}_{0}+\mathrm{C}_{1}\right)$ no sistema de cerrado mostrou que as variáveis $\mathrm{MO}$ nas profundidades estudadas e $\mathrm{P}$ e $\mathrm{K}$ na profundidade de 0,1-0,2m apresentaram dependência espacial moderada, sendo que as variáveis P e CTC na profundidade de 0,0-0,1m e K na profundidade de 0,1$0,2 \mathrm{~m}$ apresentaram dependência espacial forte (Tabela 2). No sistema de plantio direto, todas as variáveis estudadas apresentaram dependência espacial moderada. No sistema de preparo convencional, observou-se dependência espacial moderada para as variáveis $\mathrm{K}$ e CTC na profundidade de 0,0-0,1m e dependência espacial alta para o $\mathrm{P}$ nas profundidades estudadas e K e CTC na profundidade de 0,1-0,2m. Já no sistema de pastagem verificou-se dependência espacial moderada para as variáveis $K$, na profundidade de 0,0-0,1m, e CTC, nas duas profundidades estudadas, e dependência espacial forte para a MO, em ambas as profundidades estudadas, e P na camada de 0,1-0,2m.

O alcance é de fundamental importância para a interpretação dos semivariogramas, indicando a distância até onde os pontos amostrais estão correlacionados entre si (SOUZA et al., 1997; VIEIRA, 1997), ou seja, os pontos localizados em uma área cujo raio seja o alcance são mais semelhantes entre si do que os separados por distâncias maiores. Os atributos químicos apresentaram diferentes alcances, nas profundidades de 0,0-0,1m e 0,1-0,2m (Tabela 2). A variável $\mathrm{K}$ apresentou o maior alcance na profundidade de 0,0-0,1m no sistema de pastagem $(12,9 \mathrm{~m})$ e a $\mathrm{MO}$ o menor no sistema pastagem, na profundidade de 0,1 $0,2 \mathrm{~m}(4,5 \mathrm{~m})$. SOUZA et al. (1998), estudando a variabilidade espacial de atributos químicos em relação a sistemas de manejo, observaram que o sistema de plantio direto apresentou os menores alcances e o sistema de pastagem os maiores alcances. 
Tabela 2 - Modelos e parâmetros estimados dos semivariogramas experimentais para as variáveis matéria orgânica (g dm ${ }^{-3}$ ), fósforo (mg $\left.\mathrm{dm}^{-3}\right)$, potássio $\left(\mathrm{mmol}_{\mathrm{c}} \mathrm{dm}^{-3}\right)$ e capacidade de troca catiônica $\left(\mathrm{mmol}_{\mathrm{c}} \mathrm{dm}^{-3}\right)$ de amostras coletadas na malha nas profundidades de $0,0-0,1 \mathrm{~m}$ e $0,1-0,2 \mathrm{~m}$.

\begin{tabular}{|c|c|c|c|c|c|c|c|c|}
\hline \multirow{2}{*}{ Parâmetro } & \multicolumn{4}{|c|}{ Profundidade de $0,0-0,1 \mathrm{~m}$} & \multicolumn{4}{|c|}{ Profundidade de 0,1-0,2 m } \\
\hline & $\mathrm{MO}$ & $\mathrm{P}$ & K & СТС & MO & $\mathrm{P}$ & $\mathrm{K}$ & СТС \\
\hline \multicolumn{9}{|l|}{ Cerrado } \\
\hline Modelo & ${ }^{4}$ Esf & ${ }^{5} \operatorname{Exp}$ & Linear & Exp & Esf & Exp & Esf & Esf \\
\hline Efeito pepita $\left(\mathrm{C}_{0}\right)$ & 11,7 & 0,4 & 0,05 & 2,3 & 1,8 & 0,4 & 0,0003 & 3,9 \\
\hline Patamar $\left(\mathrm{C}_{0}+\mathrm{C}_{1}\right)$ & 23,4 & 1,8 & - & 47,3 & 5,1 & 0,9 & 0,009 & 10,9 \\
\hline Alcance (a) & 8,0 & 8,0 & - & 9,6 & 10,9 & 11,6 & 5,6 & 11,5 \\
\hline${ }^{1} \mathrm{C}_{0} / \mathrm{C}_{0}+\mathrm{C}_{1}$ & 50 & 22 & - & 5 & 35 & 44 & 3 & 36 \\
\hline${ }^{2} \mathrm{R}^{2}$ & 98 & 80 & 94 & 96 & 98 & 98 & 98 & 98 \\
\hline${ }^{3} \mathrm{SQR}$ & 0,8 & 0,07 & $2,0 \times 10^{-5}$ & 2,1 & 0,05 & $8,7 \times 10^{-4}$ & $1,3 \times 10^{-7}$ & $3,0 \times 10^{-3}$ \\
\hline \multicolumn{9}{|l|}{ Plantio direto } \\
\hline Modelo & Exp & Exp & Esf & Linear & Esf & Esf & Linear & Linear \\
\hline Efeito pepita $\left(\mathrm{C}_{0}\right)$ & 3,6 & 37,8 & 1,4 & 67,9 & 1,6 & 4,9 & 0,3 & 15,5 \\
\hline Patamar $\left(\mathrm{C}_{0}+\mathrm{C}_{1}\right)$ & 13,5 & 136,1 & 2,9 & - & 5,9 & 15,3 & - & - \\
\hline Alcance (a) & 5,3 & 7,2 & 10,8 & - & 12,8 & 11,7 & - & - \\
\hline $\mathrm{C}_{0} / \mathrm{C}_{0}+\mathrm{C}_{1}$ & 27 & 28 & 48 & - & 27 & 42 & - & - \\
\hline $\mathrm{R}^{2}$ & 82 & 96 & 94 & 91 & 97 & 98 & 98 & 96 \\
\hline SQR & 1,2 & 31,6 & 0,06 & 2,3 & 0,08 & 0,07 & $3,5 \times 10^{-3}$ & 8,98 \\
\hline \multicolumn{9}{|c|}{ Preparo convencional } \\
\hline Modelo & Linear & Esf & Exp & Esf & Linear & Esf & Esf & Esf \\
\hline Efeito pepita $\left(\mathrm{C}_{0}\right)$ & 0,9 & 0,6 & 0,08 & 13,5 & 2,4 & 0,32 & 2,1 & 3,8 \\
\hline Patamar $\left(\mathrm{C}_{0}+\mathrm{C}_{1}\right)$ & - & 12,1 & 0,3 & 49,3 & - & 8,32 & 9,9 & 17,2 \\
\hline Alcance (a) & - & 5,3 & 7,5 & 6,9 & - & 12,0 & 7,4 & 5,1 \\
\hline $\mathrm{C}_{0} / \mathrm{C}_{0}+\mathrm{C}_{1}$ & - & 5 & 27 & 28 & - & 4 & 21 & 22 \\
\hline $\mathrm{R}^{2}$ & 98 & 97 & 90 & 88 & 0,98 & 0,97 & 98 & 93 \\
\hline SQR & 0,2 & 0,5 & $4,5 \times 10^{-4}$ & 12,8 & 0,3 & 0,2 & 0,2 & 1,6 \\
\hline \multicolumn{9}{|l|}{ Pastagem } \\
\hline Modelo & Esf & Linear & Esf & Exp & Esf & Esf & ${ }^{6} \mathrm{EPP}$ & Esf \\
\hline Efeito pepita $\left(\mathrm{C}_{0}\right)$ & 0,8 & 8,5 & 1,2 & 2,7 & 1,7 & 1,4 & - & 4,2 \\
\hline Patamar $\left(\mathrm{C}_{0}+\mathrm{C}_{1}\right)$ & 3,8 & - & 3,0 & 10,4 & 7,8 & 6,6 & - & 8,9 \\
\hline Alcance (a) & 4,7 & - & 12,9 & 5,5 & 4,5 & 12,6 & - & 8,5 \\
\hline $\mathrm{C}_{0} / \mathrm{C}_{0}+\mathrm{C}_{1}$ & 21 & - & 40 & 26 & 22 & 21 & - & 47 \\
\hline $\mathrm{R}^{2}$ & 90 & 98 & 98 & 80 & 97 & 98 & - & 97 \\
\hline SQR & 0,1 & 0,7 & 0,01 & 1,3 & 0,08 & 0,07 & - & 0,3 \\
\hline
\end{tabular}

${ }^{1} \mathrm{C}_{0} /\left(\mathrm{C}_{0}+\mathrm{C}_{1}\right)$ = grau de dependência espacial; ${ }^{2} \mathrm{R}^{2}=$ coeficiente de determinação; ${ }^{3} \mathrm{SQR}=$ soma dos quadrados dos resíduos; ${ }^{4}$ Esf $=$ modelo esférico; ${ }^{5} \mathrm{Exp}=$ modelo exponencial; ${ }^{6} \mathrm{EPP}=$ efeito pepita puro.

\section{CONCLUSÕES}

As maiores variabilidades medidas por meio do coeficiente de variação foram observadas para o fósforo e potássio, sendo que a matéria orgânica e a CTC apresentaram coeficiente de variação médio nos diferentes usos e manejos do solo. O sistema de plantio direto apresentou acúmulo significativo de matéria orgânica, fósforo, potássio e elevação da CTC em relação aos demais sistemas estudados, além da melhoria nas condições químicas do solo. A matéria orgânica foi maior em relação ao sistema natural. As variáveis estudadas apresentaram estrutura de dependência espacial, o que permitiu o seu mapeamento, utilizando-se técnicas geoestatísticas.

\section{AGRADECIMENTOS}

À Fundação de Amparo à Pesquisa do Estado de São Paulo - FAPESP-pela concessão de bolsa de mestrado.

\section{REFERÊNCIAS}

ARAUJO, A.V. Variabilidade espacial de propriedades químicas e granulométricas do solo na definição de zonas homogêneas de manejo. 2002. 80f. Dissertação (Mestrado em do Solo) - Curso de Pós-graduação em Ciência do Solo, Universidade Estadual de São Paulo.

Ciência Rural, v.37, n.2, mar-abr, 2007. 
BAYER, C.; MIELNICZUK, J. Nitrogênio total de um solo submetido a diferentes métodos de preparo e sistemas de cultura. Revista Brasileira de Ciência do Solo, Viçosa, v.21, n.2, p.235-239, 1997.

BHATTI, A.U. et al. Estimation of soil properties and wheat yields on complex eroded hills using geostatistics and thematic mapper images. Remote Sensing Environment, New Work, v.37, n.3, p.181-191, 1991.

CAMBARDELLA, C.A. et al. Field-scale variability of soil properties in Central Iowa. Soil Science Society of American Journal, Madison, v.58, n.5, p.1501-1511, 1994.

CARVALHO, T.M. et al. Variabilidade espacial da retenção de água em um Latossolo Vermelho-Amarelo através da geoestatística. Ciência e Prática, Lavras, v.16, n.4, p.521528, 1992.

CARVALHO, O.S. et al. Variabilidade espacial de algumas propriedades químicas e físicas de um solo submetido a diferentes sucessões de cultivo. Revista Brasileira de Ciência do Solo, Viçosa, v.22, n.3, p.497-503, 1998.

CARVALHO, J.R.P. et al. Geoestatística na determinação da variabilidade espacial de características químicas do solo sob diferentes preparos. Pesquisa Agropecuária Brasileira, Brasília, v.37, n.8, p.1151-1159, 2002.

CENTURION, J.F. et al. Efeitos de sistemas de preparo nas propriedades químicas de um solo sob cerrado cultivado com soja. Revista Brasileira de Ciência do Solo, Campinas, v.9, n.3, p.267-270, 1985.

DE MARIA, I.C.; CASTRO, O.M. Fósforo, potássio e matéria orgânica em um Latossolo Roxo, sob sistemas de manejo com milho e soja. Revista Brasileira de Ciência do Solo, Campinas, v.17, n.4, p.471-477, 1993.

DERPSCH, R. et al. Controle de erosão no Paraná, Brasil: sistemas de cobertura do solo, plantio direto e preparo conservacionista do solo. Eschborn, República Federal da Alemanha: Sonderpublikation der GTZ/IAPAR, 1991. 272p. (Boletim, 145).

ELTZ, F.L.F. et al. Efeito de sistemas de preparo do solo nas propriedades físicas e químicas de um Latossolo Bruno álico. Revista Brasileira de Ciência do Solo, Campinas, v.13, n.2, p.259-267, 1989.

ISAAKS, E.H.; SRIVASTAVA, R.M. An introduction to applied geoestatistics. New York: Oxford University, 1989. 561p.

McBRATNEY, A.B.; WEBSTER, R. Choosing functions for semi-variograms of soil properties and fitting them to sampling estimates. Journal Soil Science, Oxford, v.37, n.3, p.617639, 1986.

OLIVEIRA, J.J. et al. Variabilidade espacial de propriedades químicas em um solo salino-sódico. Revista Brasileira de Ciência do Solo, Viçosa, v.23, n.4, p.783-789, 1999.

OVALLES, F.; REY, J. Variabilidad interna de unidades de fertilidad en suelos de la depresión del Lago de Valencia. Agronomía Tropical, Madrid, v.44, n.1, p.41-65, 1994.

RAIJ, B. van et al. (Ed). Análise química para avaliação da fertilidade de solos tropicais. Campinas: Instituto Agronômico, 2001. 285p.
ROBERTSON, G.P. GS : geostatistics for the environmental sciences (version 5.1 for windows). Plainwell: Michigan, Gamma Design Software, 1998. 152p.

SALVIANO, A.A.C. et al. Variabilidade espacial de atributos de solo e de Crotalaria juncea $(L)$ em área severamente erodida. Revista Brasileira de Ciência do Solo, Viçosa, v.22, n.1, p.115-122, 1998.

SILVEIRA, P.M. et al. Amostragem e variabilidade especial de características químicas de um latossolo submetido a diferentes sistemas de preparo. Pesquisa Agropecuária Brasileira, Brasília, v.35, n.10, p.2057-2064, 2000.

SILVEIRA, P.M.; STONE, L.F. Teores de nutrientes e matéria orgânica afetados pela rotação de cultura e sistema de preparo do solo. Revista Brasileira de Ciência do Solo, Viçosa, v.25, n.3, p.387-394, 2001.

SOUZA, L.S. et al. Variabilidade de propriedades físicas e químicas do solo em um pomar cítrico. Revista Brasileira de Ciência do Solo, Viçosa, v.21, n.3, p.367-372, 1997.

SOUZA, L.S. et al. Variabilidade de fósforo, potássio e matéria orgânica no solo, em relação a sistemas de manejo. Revista Brasileira de Ciência do Solo, Viçosa, v.22, n.1, p.77-86, 1998.

SOUZA, Z.M.; ALVES, M.C. Propriedades químicas de um Latossolo Vermelho distrófico de cerrado sob diferentes usos e manejos. Revista Brasileira de Ciência do Solo, Viçosa, v.27, n.1, p.133-139, 2003.

SOUZA, C.K. et al. Influência do relevo na variação anisotrópica dos atributos químicos e granulométricos de uma latossolo em Jaboticabal-SP. Engenharia Agrícola, Jaboticabal, v.23, n.3, p.486-495, 2003.

SCHLOTZHAVER, S.D.; LITTELL, R.C. SAS: system for elementary statistical analysis. 2.ed. Cary, 1997. 905p.

TRANGMAR, B.B. et al. Applications of geostatistics to spatial studies of soil properties. Advances in Agronomy, San Diego, v.38, n.1, p.45-94, 1985.

TSCHIEDEL, M.; FERREIRA, M.F. Introdução à agricultura de precisão: conceitos e vantagens. Ciência Rural, Santa Maria, v.32, n.1, p.159-163, 2002.

VIEIRA, S.R. et al. Geoestatiscal theory and application to variability of some agronomical properties. Hilgardia, Oakland, v.51, n.1, p.1-75, 1983.

VIEIRA, S.R. Variabilidade espacial de argila, silte e atributos químicos em uma parcela experimental de um Latossolo Roxo de Campinas (SP). Bragantia, Campinas, v.56, n.1, p.18190, 1997.

WARRICK, A.W.; NIELSEN, D.R. Spatial variability of soil physical properties in the field. In: HILLEL, D. (Ed). Applications of soil physics. New York: Academic, 1980. Cap.2, p.319-344.

WILDING, L.P.; DREES, L.R. Spatial variability and pedology. In: WINDING, L.P. Pedogenesis and soil taxonomy: I concepts and interactions. Amsterdan: Elsevier, 1983. p.83116 . 